Article

\title{
Water-in-Water Emulsion as a New Approach to Produce Mesalamine-Loaded Xylan-Based Microparticles
}

\author{
Bartolomeu S. Souza ${ }^{1}$, Henrique R. Marcelino ${ }^{2}$ (D) Francisco Alexandrino, Jr. ${ }^{3}$ (D), \\ Silvana C. C. Urtiga ${ }^{1}$, Karen C. H. Silva ${ }^{4}$, Daniel C. F. Soares ${ }^{5}$ and Eryvaldo S. T. Egito ${ }^{1,3, *}$ (1) \\ 1 Programa de Pós-graduação em Ciências da Saúde, Universidade Federal do Rio grande do Norte, \\ Natal/RN 59012-570, Brazil \\ 2 Departamento do Medicamento, Universidade Federal da Bahia, Salvador/BA 40170-115, Brazil \\ 3 Programa de Pós-graduação em Nanotecnologia Farmacêutica, Universidade Federal do Rio Grande do \\ Norte, Natal/RN 59012-570, Brazil \\ 4 Programa de Pós-graduação em Ciências Farmacêuticas, Universidade Federal do Rio Grande do Norte, \\ Natal/RN 59012-70, Brazil \\ 5 Universidade Federal de Itajubá-campus Itabira-Avenida Irma Ivone Drumond, Itabira/MG 35903-087, Brazil \\ * Correspondence: socratesegito@gmail.com; Tel.: +55-84-3342-9817
}

Received: 27 June 2019; Accepted: 31 July 2019; Published: 27 August 2019

\section{Featured Application: Controlled Release of Drugs.}

\begin{abstract}
The water-in-water emulsion method has been reported as a technique able to prepare microparticles without using harmful solvents. However, there are few reports showing the encapsulation of small molecules into microparticles produced within this technique. The probable reason relays on the rapid diffusion of these molecules from the discontinuous phase to the continuous phase. In the present study, xylan microparticles containing mesalamine were produced and the doubled crosslinking approach, used to promote higher encapsulation rates, was disclosed. To achieve this goal, a $2^{3}$ full factorial design was carried out. The results revealed that all formulations presented spherical-shaped microparticles. However, at specific conditions, only few formulations reached up to $50 \%$ of drug loading. In addition, the new xylan-based microparticles formulation retained almost $40 \%$ of its drug content after $12 \mathrm{~h}$ of a dissolution assay likely due to the degree of crosslinking. Thus, the doubled crosslinking approach used was effective on the encapsulation of mesalamine and may pave the way to successfully produce other polysaccharide-based carriers for clinical use.
\end{abstract}

Keywords: xylan; water-in-water emulsion; mesalamine; mathematical modeling

\section{Introduction}

The preparation of microparticles based on hydrophilic polymers, such as polysaccharides derivatives, using techniques based on emulsion templates, majorly requires the use of organic solvents in order to form the hydrophilic and hydrophobic phases in which the internal hydrophilic phase contains the polymer [1-3]. In addition, together with the use of crosslinkers such as glutaraldehyde and terephthaloyl chloride, the microparticles produced by this way may present an important degree of toxicity [1,4].

Xylan, which is the second most abundant polysaccharide of the biosphere, is mainly presented in hardwood and perennial plants such as grasses, cereals, and herbs [5]. The use of xylan for biomedical applications is based on its important biocompatibility and selective degradation in the gastrointestinal 
tract, which occurs in the colon through enzymatic hydrolysis by the microbiota. Our group has lately exploited this feature in order to develop colon-specific drug delivery systems [6-8].

As previously mentioned, the preparation of polysaccharide-based microparticles, including xylan-based microparticles (XBM), mostly requires the use of organic/harmful solvents such as cyclohexane, and crosslinkers, such as terephthaloyl chloride, during their preparation $[1,9,10]$. On this behalf, the water-in-water emulsion approach can produce microparticles by the mixture of two immiscible aqueous polymeric solutions. Once these solutions are mixed, there is a formation of a system with a positive Gibbs free energy $(\Delta G)$ (Equation (1)).

$$
\Delta G=\Delta H_{\text {mix }}-\mathrm{T} \Delta S_{\text {mix }}
$$

This phenomenon happens due to the high molecular weight of the polyethylene glycol (PEG), which limited the translational motion of the polymeric chains. This results in a smaller value of $\Delta S_{\text {mix }}$ than $\Delta H_{\text {mix }}$. Hence, it results in a positive $\Delta G$, and, therefore, leads to phase separation [11]. Similarly to the traditional oil-in-water and water-in-oil emulsions, these two polymeric phases, once homogenized, may form two different phases, which include a continuous and a discontinuous one. Thus, once the two phases are obtained and a crosslinker is added to the dispersion, particles can be obtained [12,13].

The reports about microparticles prepared by the water-in-water emulsion technique hardly ever describe the encapsulation of active molecules [14-16]. Nonetheless, when tried, the encapsulated molecules are mostly biomolecules, such as proteins and peptides [17]. A probable explanation for such a phenomenon would be related to the free mobility of the small hydrophilic molecules into the dispersion. Once both phases are aqueous, the encapsulation efficiency is hindered [14].

The preparation of microparticles loaded with small active molecules through the water-in-water emulsion technique has been a pharmaceutical technology-based challenge. In this sense, in the present work, xylan microparticles loaded with mesalamine (5-ASA), which is a low molecular weight molecule, were prepared through a water-in-water emulsification process using different crosslinking approaches, designed through a $2^{3}$ full factorial design with a central point.

\section{Materials and Methods}

\subsection{Materials}

Xylan from beech wood, 5-ASA, and trisodium trimetaphosphate (STMP) were purchased from Sigma-Aldrich ${ }^{\circledR}$ (São Paulo, Brazil). Polyethylene glycol (PEG, MW = 20,000 Da) came from Merck $^{\circledR}$ (São Paulo, Brazil). Potassium chloride was purchased from QEEL ${ }^{\circledR}$ (São Paulo, Brazil). Sodium chloride, sodium hydroxide, calcium chloride, and sodium phosphate dibasic anhydrous were purchased from Vetec ${ }^{\circledR}$ Chemical (Rio de Janeiro, Brazil). Anhydrous monobasic potassium phosphate was from ISOFAR ${ }^{\circledR}$ (Rio de Janeiro, Brazil) and ethanol 98\% ${ }_{(\mathrm{v} / \mathrm{v})}$ from Atriom ${ }^{\circledR}$ (São Paulo, Brazil). Water (conductivity $\leq 3.5 \mu \mathrm{S} / \mathrm{cm}$ ), obtained from deionization and followed by a reverse osmosis process, was used to produce the microparticles and prepare the dissolution medium. All chemicals were of an analytical grade and used as received.

\subsection{Phase Diagram of Xylan and PEG}

To evaluate the concentrations of xylan and PEG required to achieve the formation of water-in-water emulsions, a phase diagram was obtained from acquired data in different experiments. First, a stock solution containing $35 \%(\mathrm{w} / \mathrm{v})$ of xylan and another with $32 \%(\mathrm{w} / \mathrm{v})$ of PEG were prepared. Afterward, successive dilutions were made using $1 \mathrm{~N} \mathrm{NaOH}$ and water, to reach a concentration range from $0.1 \%_{(\mathrm{w} / \mathrm{v})}$ to $32 \%_{(\mathrm{w} / \mathrm{v})}$ for xylan and from $0.5 \%_{(\mathrm{w} / \mathrm{v})}$ to $29 \%_{(\mathrm{w} / \mathrm{v})}$ for PEG, respectively. These solutions were brought together in a 1:4(v/v) ratio and the phase separation was evaluated by the naked eye [16]. 


\subsection{Production of the 5-ASA-Loaded XBM}

The 5-ASA-loaded XBM was prepared from an emulsion obtained by the previously mentioned phase diagram. The microparticles were produced following the flow chart presented in Figure 1. First, a solution containing $4 \%(\mathrm{w} / \mathrm{v})$ of xylan, $1 \%_{(\mathrm{w} / \mathrm{v})}$ of STMP, and $0.05 \%_{(\mathrm{w} / \mathrm{v})}$ of 5 -ASA was prepared. Simultaneously, a PEG solution containing $32 \%(\mathrm{w} / \mathrm{v})$, with or without $\mathrm{CaCl}_{2}$, was also prepared. In the sequence, the xylan solution was slowly poured into the PEG solution to reach a proportion of $1: 4_{(\mathrm{v} / \mathrm{v})}$, respectively. The dispersion was kept under constant magnetic stirring for $5 \mathrm{~min}$ and, then, incubated at $45^{\circ} \mathrm{C}$ for $6 \mathrm{~h} \mathrm{[10].} \mathrm{Afterward,} \mathrm{to} \mathrm{cease} \mathrm{the} \mathrm{reaction} \mathrm{and} \mathrm{lead} \mathrm{to} \mathrm{microparticles} \mathrm{precipitation,} \mathrm{ethanol}$ $98 \%(\mathrm{v} / \mathrm{v})$ was added to the mixture. Then, washing steps were carried out by centrifugation $(2136 \mathrm{~g}$ for $5 \mathrm{~min})$ with ethanol $98 \%(\mathrm{v} / \mathrm{v})$ and deionized water. Lastly, the 5-ASA-loaded XBM were dried at room temperature and stored at $25^{\circ} \mathrm{C}$.

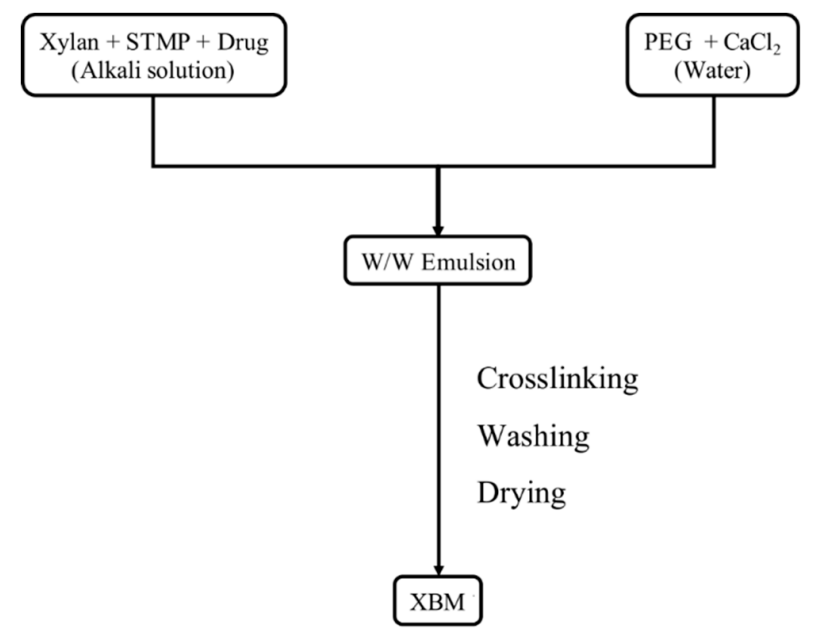

Figure 1. Flow chart for the production of the xylan-based microparticles $(\mathrm{XBM})$ and XBM-Ca. STMP = Sodium trimethaphosphate. $\mathrm{PEG}=$ Polyethylene glycol. $\mathrm{MW}=20,000 . \mathrm{CaCl}_{2}=$ Calcium chloride.

\subsection{Full Factorial Design}

In order to improve the drug encapsulation into the XBM, a $2^{3}$ full factorial design with a center point was built (Table 1). The concentrations of xylan, $\mathrm{CaCl}_{2}$, and STMP were tested according to Table 1, while $5.0 \mathrm{mg}$ of 5-ASA was added in all formulations (XBM-Ca-F1-XBM-Ca-F9). Afterward, a mathematical modeling of the data was carried out in order to determine the parameters that had higher influence over the encapsulation efficiency.

Table 1. Experimental results of average diameter size and EE\% of XBM and all XBM-Ca prepared based on the $2^{3}$ experimental design.

\begin{tabular}{cccccc}
\hline Samples & Xylan (\%) & STMP (\%) & $\mathbf{C a C l}_{\mathbf{2}} \mathbf{( \% )}$ & Average Size $\boldsymbol{\mu m} \pm \mathbf{( S D}$ *) & EE (\%) $\pm($ SD) \\
\hline XBM & 4.0 & 0.5 & - & $10.7 \pm 2.0$ & $3.6 \pm 2.5$ \\
XBM-Ca-F1 & 5.0 & 0.5 & 0.5 & $4.6 \pm 0.1$ & $29.1 \pm 2.3$ \\
XBM-Ca-F2 & 5.0 & 1.0 & 0.5 & $4.6 \pm 0.1$ & $16.4 \pm 6.2$ \\
XBM-Ca-F3 & 2.0 & 0.5 & 0.5 & $6.1 \pm 0.4$ & $20.9 \pm 3.6$ \\
XBM-Ca-F4 & 5.0 & 1.0 & 2.0 & $5.1 \pm 1.1$ & $35.3 \pm 9.3$ \\
XBM-Ca-F5 & 2.0 & 1.0 & 0.5 & $6.1 \pm 0.4$ & $32.6 \pm 5.5$ \\
XBM-Ca-F6 & 3.5 & 0.75 & 1.25 & $6.3 \pm 0.2$ & $32.6 \pm 0.8$ \\
XBM-Ca-F7 & 5.0 & 0.5 & 2.0 & $5.6 \pm 0.2$ & $35.4 \pm 12.3$ \\
XBM-Ca-F8 & 2.0 & 0.5 & 2.0 & $4.5 \pm 0.1$ & $29.8 \pm 8.8$ \\
XBM-Ca-F9 & 2.0 & 1.0 & 2.0 & $4.8 \pm 0.1$ & $49.6 \pm 0.5$ \\
\hline
\end{tabular}

$\mathrm{STMP}=$ trisodium trimetaphosphate. $\mathrm{CaCl}_{2}=$ calcium chloride. $\mathrm{XBM}=$ xylan-based microparticles. $\mathrm{XBM}-\mathrm{Ca}=\mathrm{XBM}$ also crosslinked with calcium chloride. $\mathrm{EE} \%$ = encapsulation efficiency. $\mathrm{F}=$ Formulation. The PEG (polyethylene glycol) concentration remained constant in all formulations $\left(32 \%(\mathrm{w} / \mathrm{v})\right.$ ). ${ }^{*}$ This standard deviation is from the average size obtained during the analysis of 1500 particles (3 times 500), and not from the particle size distribution. 


\subsection{Morphologic Analyses and Particle Size Distribution}

The morphology and particle size distribution were evaluated by optical microscopy, using two optical microscopes, Model TM 800 (Taimin ${ }^{\circledR}$, João Pessoa, Brazil) and Model 020507.010 (Leica ${ }^{\circledR}$, Bellevue, WA USA) at a magnification of $10 x$. In order to enhance the contrast of the particles during the analyses, several droplets of methylene blue solution $0.01 \%(w / v)$ were used to re-suspend the microparticles. For the measurement of the particle size distribution, the powder of the microparticles was placed onto glass slides followed by the counting of 1500 particles $(3 \times 500)$. The particle size of each formulation was assessed, according to the Feret's diameter [8]. Then, the acquired data were plotted using the Prism ${ }^{\circledR}$ (Version 5.03, GraphPad ${ }^{\circledR}$, San Diego, CA, USA).

\subsection{Drug Loading Efficiency (\%)}

The amount of 5-ASA encapsulated into the microparticles was evaluated through an UV/Vis Spectrophotometry technique (Biochrom ${ }^{\circledR}$ Libra ${ }^{\circledR}$ S32 UV/Vis, Cambridge, UK), at $\lambda=322 \mathrm{~nm}$, with the following parameters: $y=27.87 x-0.0303, R^{2}=0.999$, and relative standard deviation below $0.05 \%$ for all data points, in which it was demonstrated that the microparticles shell did not interfere. The microparticles content was released, using $1 \mathrm{~N} \mathrm{NaOH}$ and an ultrasound probe (13 $\mathrm{mm}$ probe, Vibra-cell ${ }^{\circledR}$ 75041, Fischer Bioblock Scientific, Aalst, Belgium). In addition, 3 mg of the microparticles powder was immersed into $3 \mathrm{~mL}$ of $1 \mathrm{~N} \mathrm{NaOH}$ solution. Then, the mixture was kept at $37^{\circ} \mathrm{C}$ under orbital stirring at $100 \mathrm{rpm}$, overnight. Afterward, the suspension was sonicated with an amplitude of $40 \%$ and $20 \mathrm{KHz}$ for $1 \mathrm{~min}$ and centrifuged at $2136 \mathrm{~g}$ for $5 \mathrm{~min}$. The amount of 5-ASA loaded was determined in the supernatant, and measured at $\lambda=322 \mathrm{~nm}$, which followed the previously validated spectrophotometric method. The drug loading efficiency $(\mathrm{EE} \%)$ was calculated by the following equation.

Encapsulation efficiency $(\%)=($ quantified drug content/theoretical drug content $) \times 100$

\subsection{Attenuated Total Reflectance Infrared (ATR-IR) Spectroscopy Analysis}

The interaction between the xylan, the 5-ASA, and the STMP during the crosslinking process was evaluated by ATR-IR spectroscopy (Spectrum 65, Waltham, MA, USA). The ATR-IR spectroscopy measurements were performed using the samples on the solid state. The samples were placed on the crystal area and the pressure arm was positioned over the crystal/sample area. Each sample was subjected to four scans at $1 \mathrm{~cm}^{-1}$ resolution at room temperature using acetone to clean the crystal between the samples. The runs were carried out from the range of 4000 to $500 \mathrm{~cm}^{-1}$. Once the analyses were performed, the graphs were plotted using OriginPro ${ }^{\circledR}$ (Version 2015, Northampton, MA, USA).

\subsection{X-ray Diffraction (XRD)}

XRD analysis was performed for the 5-ASA and xylan alone, and the formulation XBM-Ca-F9 with and without the 5-ASA. X-ray scattering angle measurements were performed with a copper anode radiation $\mathrm{K} \alpha(\lambda=0.15418 \mathrm{~nm}, 40 \mathrm{kV}, 20 \mathrm{~mA})$ attached to the diffractometer (Bruker, model D8 Advance, Karlsruhe, Germany). A scan rate of $2^{\circ}$ /minute across the range of $20^{\circ}$ to $80^{\circ} 2 \theta$ was used to determine each spectrum.

\subsection{In Vitro Drug Release}

Initially, $10 \mathrm{mg}$ of XBM-Ca-F9 was added in $30 \mathrm{~mL}$ of phosphate buffered saline $\mathrm{pH}=7.4$ at $37^{\circ} \mathrm{C}$. Subsequently, the recipients were sealed and maintained under orbital stirring of $100 \mathrm{rpm}$, and at specific times $(0.5,1,2,3,4,5,6$, and $12 \mathrm{~h})$, aliquots of $5 \mathrm{~mL}$ were withdrawn from the recipients and centrifuged at $2136 \mathrm{~g}$ for $5 \mathrm{~min}$. Five $\mathrm{mL}$ of fresh media were added in order to maintain the sink condition. Thereafter, the amount of 5-ASA in the supernatant was determined through UV/Vis Spectrophotometry (Biochrom ${ }^{\circledR}$ Libra $^{\circledR}$ S32 UV/Vis, Cambridge, UK). The amount of 5-ASA released 
was calculated and expressed as the accumulative percentage of the drug released versus time through the Prism ${ }^{\circledR}$ (Version 5.03, GraphPad ${ }^{\circledR}$, San Diego, CA, USA). After $12 \mathrm{~h}$, the amount of 5-ASA remaining into the microparticles was analyzed following the procedure described in Section 2.6, after incubation at $37^{\circ} \mathrm{C}$ for $2 \mathrm{~h}$.

\subsection{Mathematical Modeling of the In Vitro 5-ASA Release}

The mathematical modeling of the data from the drug release assay was performed using the Add-in DDsolver for Microsoft ${ }^{\circledR}$ Excel [18] in order to describe the mechanism by which the 5-ASA was released from the microparticles. The choice of the model that best fitted the experimental data was based on the adjusted coefficient of determination (adjusted- $\mathrm{R}^{2}$ ), which corresponds to the adjustment of the theoretical models to the experimentally obtained data. Additionally, the Root Average Square Error (RMSE) was used to evaluate the difference between the values obtained experimentally and those predicted by the model. Therefore, the model that best describes the experimental data was the one showing the largest adjusted- $\mathrm{R}^{2}$ and the lower RMSE [18].

\subsection{Statistical Analysis}

The experimental design was performed in duplicate. Results were presented as average \pm standard deviation (SD) of two independent analyses. The analysis of variance (ANOVA) was performed using the STATISTICA ${ }^{\circledR}$ (Version 10, StatSoft, Palo Alto, CA, USA). P-values lower than 0.05 were assumed to be statistically significant.

\section{Results and Discussion}

\subsection{Phase Diagram of Xylan and PEG}

Water-in-water emulsions can be obtained by mixing two aqueous solutions of hydrophilic polymers. A biphasic system only is obtained when a certain polymer concentration is reached. These emulsions recently have been granted important attention due to the great potential applications in food and cosmetics preparations. In this sense, the achievement of a monophasic dispersion is mandatory [19]. The water-in-water emulsification process allows obtaining microparticles with narrow size distribution loaded with an active compound by avoiding the use of organic solvents and surfactant agents [11]. Stenekes and Hennink, still in the 1990s, showed that the average size of the particles prepared using the water-in-water emulsion method depends on the volume ratio of the discontinuous/continuous phase, the viscosity of the solutions, and the molecular weight of the polymers [20].

On the other hand, the microencapsulation into xylan-based microparticles of low molecular weight molecules, such as 5-ASA, has been a pharmaceutical technology challenge due to the restricted microparticles' physicochemical stability and the use of harmful solvents to produce them [10]. In this regard, water-in-water emulsion systems can solve all drawbacks to produce such xylan-based microparticles. The phase diagram is the more common approach used to develop water-in-water emulsions [11]. Using such an approach, it is not only possible to define the region of the phase separation, but also to control the size of the internal phase domains, which will be further used as a template to produce the microparticles.

In this regard, the phase diagram prepared with PEG and xylan solutions was obtained and the results are available in Figure 2. The obtained data revealed the potential templates for the preparation of XBM. The region that shows the different and stable combinations between PEG and xylan are identified by the points above the tie-line (solid line, Figure 2). Located at this region, the emulsion constituted by PEG and xylan, at the concentration of $32 \%(\mathrm{w} / \mathrm{v})$ and $4 \%_{(\mathrm{w} / \mathrm{v})}$, respectively, was further used due to the clear formation of droplets at the micro-scale range (data not shown). 


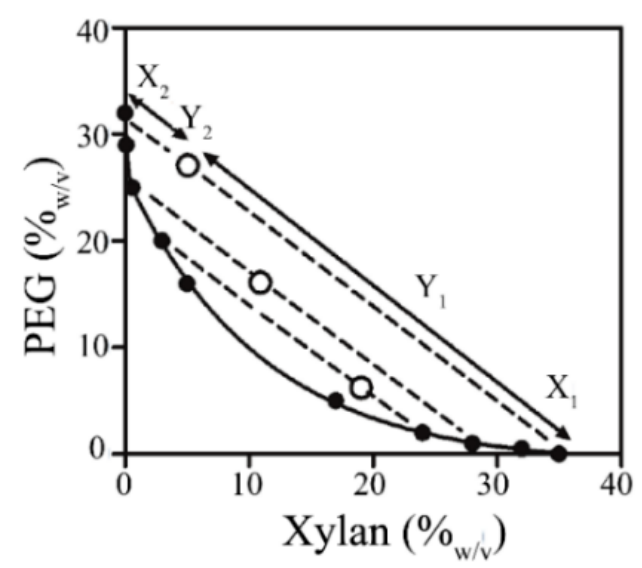

Figure 2. Phase diagram of xylan and PEG solutions. The solid line (-) represents the binodal line and the dashed lines (- - ) or the tie-lines. Below the binodal lines, the systems have just one phase, and, above it, the open circle $(\bigcirc)$ represents the point at which the phase separation occurs. $X_{1}$ stands for the xylan dispersion at $40 \%{ }_{(w / v)}, X_{2}$ is the PEG enriched solution $32 \%(w / v)$, and $Y_{1}$ and $Y_{2}$ represent the volume ratio of the two phases for the formation of the emulsions.

\subsection{Production and Characterization of XBM}

The production of the XBM using the template chosen through the phase diagram was successfully achieved by the addition of STMP, as a cross-linker, and the incubation of the mixture at $45^{\circ} \mathrm{C}$ for $6 \mathrm{~h}$. From the macroscopic point of view, after stirring the two solutions, the emulsion presented a yellowish color and a homogeneous appearance. Once 5-ASA was added, samples also remained homogeneous, but reddish in tone. It is noteworthy that all formulations did not show phase separation after the incubation period. In addition, the XBM formulations had about a $35 \%$ yield, while the formulations containing $\mathrm{CaCl}_{2}$ showed about a $60 \%$ yield. Thus, this parameter was the first evidence of the rapid formation of the microparticles pulling together xylan chains and hindering their diffusion to the continuous phase. Microscopically, the XBM formulation exhibited non-aggregated spherical microparticles (Figure 3A), with a homogeneous particle population distribution, and an average diameter of $10.7 \pm 2.0 \mu \mathrm{m}$ (Figure 3C).

The encapsulation efficiency of the 5-ASA was below $4 \%$ for the XBM formulation (Table 1). The probable reason for such a low encapsulation rate might be due to the diffusion of the 5-ASA to the continuous phase. This phenomenon might be the consequence of the use of the $1 \mathrm{~N} \mathrm{NaOH}$ on the production of the xylan solution and a long time to achieve the crosslinking process. The alkaline solution increases the $\mathrm{pH}$ of the emulsion to approximately 12 and allows the 5-ASA to be soluble on both phases of the emulsion and the crosslinking process lasts for around $6 \mathrm{~h}$. In fact, the 5-ASA is largely soluble into alkaline solutions while its solubility in water is $1.41 \mathrm{mg} / \mathrm{mL}$ at $37^{\circ} \mathrm{C}[21,22]$. 


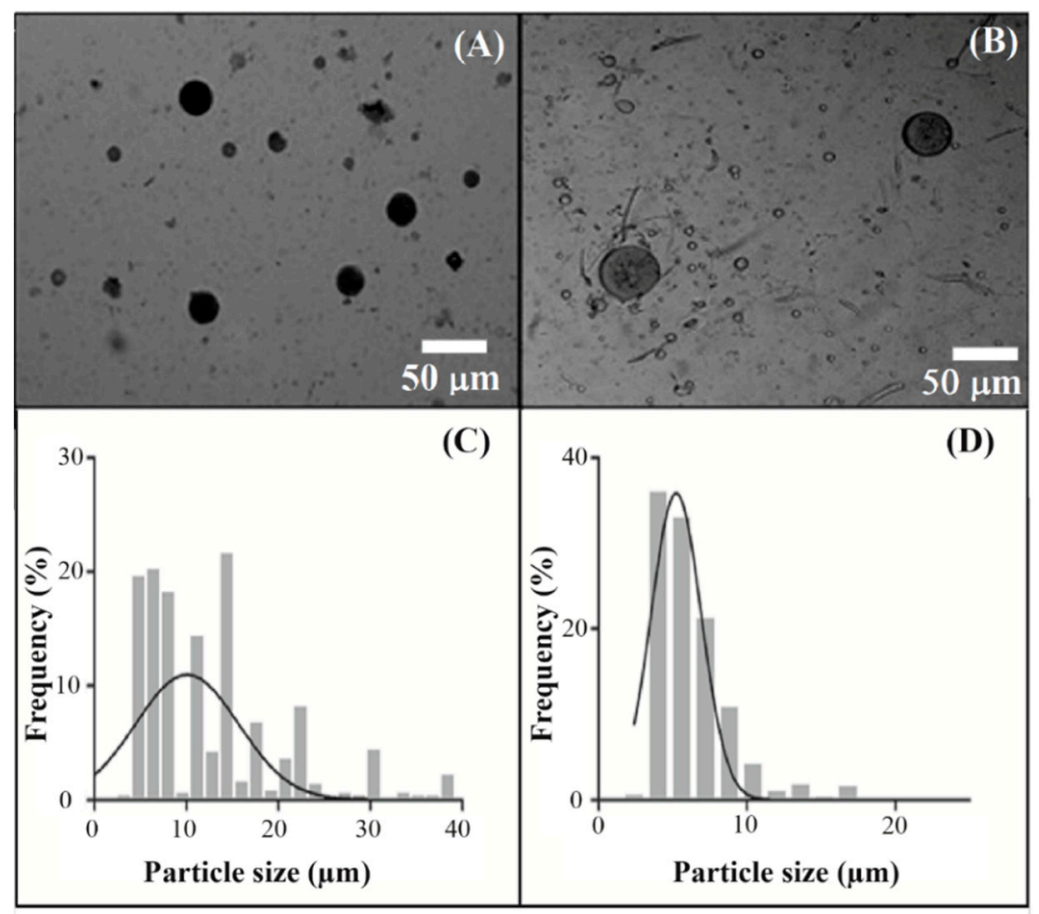

Figure 3. Optical microscopy (A,B) and particle size distribution $(\mathbf{C}, \mathbf{D})$ of $\mathrm{XBM}(\mathbf{A}, \mathbf{C}$, respectively) and XBM-Ca-F9 (B,D, respectively), at a magnification of $10 x$ with their respective Gaussian distribution (bold line, C,D).

\subsection{Influence of the $\mathrm{CaCl}_{2}$ in the 5-ASA Loading}

In order to increase the drug encapsulation into the XBM and improve its hardness, the addition of $\mathrm{CaCl}_{2}$ during the microparticles production was used as a strategy to promote a faster crosslinking within the xylan chains. This approach could prevent the diffusion of the 5-ASA within the continuous phase, while hardening the xylan droplet. Simultaneously, the covalent chemical crosslinking between STMP and xylan would happen during the incubation period. In addition, the $\mathrm{CaCl}_{2}$ might perform a role as a crosslinker, on the sites that would not be occupied by the STMP, which may increase the number of interaction points along the polymeric chains (Figure 4). Consequently, the crosslinking and compactness of the produced microparticles would also increase, which can be observed by a reduction on the average size on the formulations containing $\mathrm{CaCl}_{2}$ (XBM-Ca-F1 to XBM-Ca-F9) compared to the ones without $\mathrm{CaCl}_{2}$ (XBM) (Table 1) [23].

Similarly, based on XBM, the optical microscopy of the formulation XBM-Ca-F9 (Figure 3B) shows not only a spherical shape and absence of aggregates, but also a uniform distribution particle size (Figure 3D). In addition, the $\mathrm{CaCl}_{2}$ promoted a reduction of approximately $50 \%$ on the average diameter of the formulation, from 10.70 (for XBM) to $4.77 \mu \mathrm{m}$ (for XBM-Ca-F9). As can be seen in the formulations XBM-Ca-F4 and XBM-Ca-F6 (Table 1), the increase of $\mathrm{CaCl}_{2}$ to $2 \%(\mathrm{w} / \mathrm{v})$ promoted a decrease in the average particle size.

The factorial design analysis showed that only the main effect of $\mathrm{CaCl}_{2}$ and the xylan-STMP interaction were statistically significant to increase the 5-ASA encapsulation into XBM ( $p$-value $<0.05)$. As shown in Figure 5, the increment of the $\mathrm{CaCl}_{2}$ improved the encapsulation of 5-ASA into the microparticles. This phenomenon was related to the faster crosslinking process of the $\mathrm{Ca}^{2+}$, mainly on the surface of the droplet, when compared to the STMP alone. The $\mathrm{Ca}^{2+}$ could be able to keep the drug entrapped while the network between xylan an STMP was formed [24]. On the other hand, the interaction between xylan and STMP has a negative impact on the 5-ASA encapsulation. It is likely that the amount of STMP used for the crosslinking reactions was insufficient to tightly link the xylan chains. 
Previous experiments showed that the ideal condition to produce XBM was the use of $1 \%{ }_{(w / v)}$ of STMP. At high polymer concentrations, the formation of microparticles was hindered (data not shown).

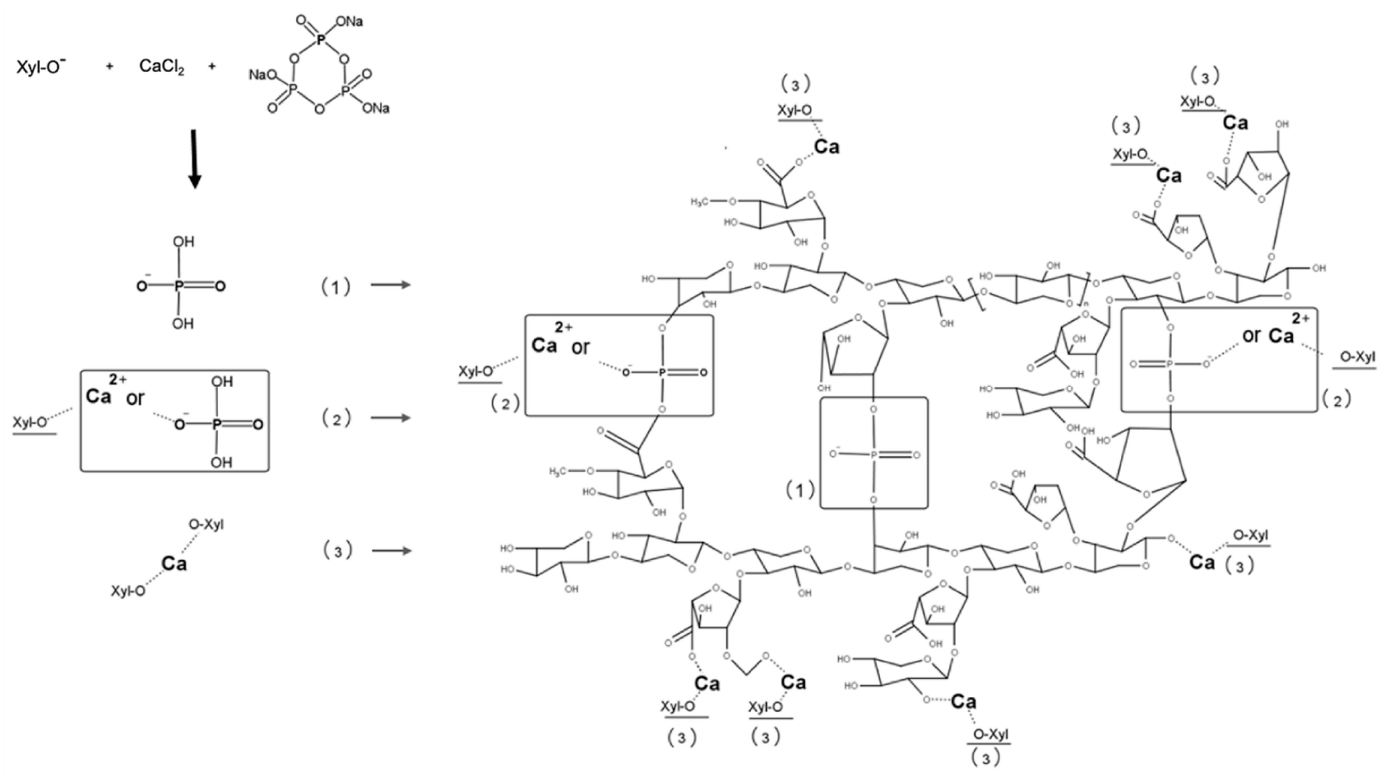

Figure 4. Scheme of the crosslinking reaction between xylan (Xyl), $\mathrm{CaCl}_{2}$, and STMP. Reaction (1) represents only the chemical bond between STMP-Xyl. Reaction (2) represents the process of chemical crosslinking between $\mathrm{Ca}^{2+}-\mathrm{Xyl}-\mathrm{STMP}$, respectively, and Reaction (3) represents the ionic interaction between $\mathrm{Ca}^{2+}-\mathrm{Xyl}$.

(A)

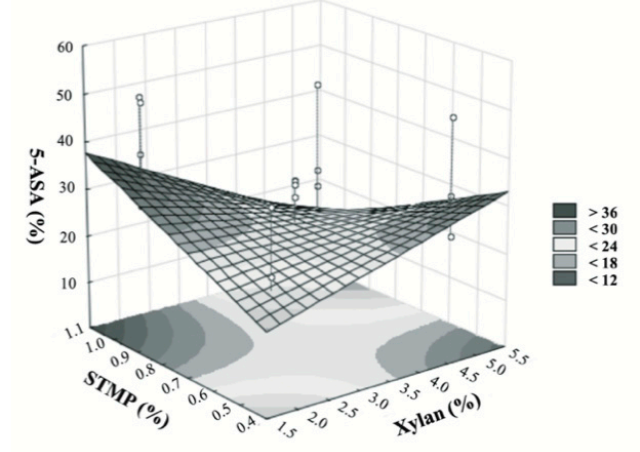

(B)

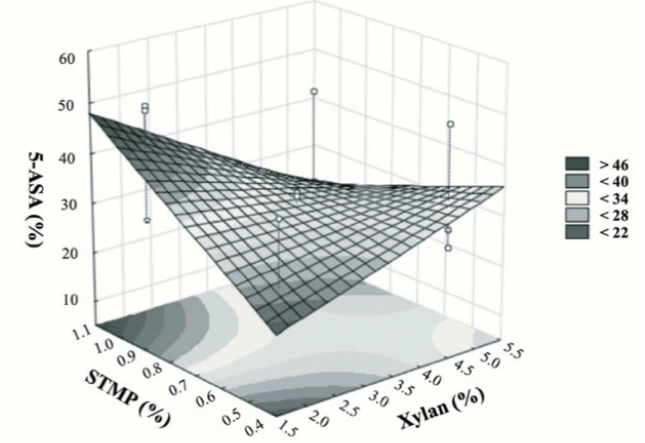

(C)

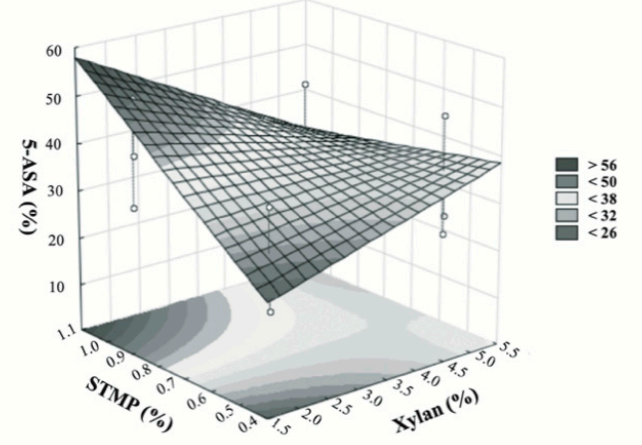

Figure 5. Response surface plot. Correlation of the encapsulation of 5-ASA with xylan-STMP and $\mathrm{CaCl}_{2}$ concentrations into the formulation. The STMP and xylan concentration were maintained (x-axis), while the concentration of $\mathrm{CaCl}_{2}$ was increased. In (A) it was used $0.5 \%(\mathrm{w} / \mathrm{v})$ of $\mathrm{CaCl}_{2}$, in (B) $1.25 \%_{(\mathrm{w} / \mathrm{v})}$ and in $(\mathrm{C}) 2 \%(\mathrm{w} / \mathrm{v})$. 
Additionally, the encapsulation of 5-ASA was enhanced at the lower level of xylan and at the higher level of STMP (Figure 5A-C). The particles formed within these concentrations likely have a smaller pore size due to the high crosslinking degree, which, consequently, improves the encapsulation of 5-ASA [25].

From the mathematical modeling of the factorial design experimental data, it was possible to retrieve a predictive model with an adjusted- $R^{2}=0.4661$ and a predictive- $R^{2}=0.1253$. This large difference might be justified by the standard deviation obtained from some formulations, such as XBM-Ca-F4, XBM-Ca-F7, and XBM-Ca-F8. However, all these formulations showed a high xylan-STMP mass ratio, 5:1, 10:1, and 4:1, respectively, while the optimal formulation (XBM-Ca-F9) had a xylan-STMP mass ratio of 2:1 (Table 1, Figure 5). This data also corroborates to the ones from the literature, in which it could be observed that the crosslinking reaction was important not only to the encapsulation of the drug into the microparticles, but also to the control of their release to the media (buffers and physiological fluids) $[23,24]$. Recently, xylan-based microspheres were prepared by an emulsion-template technique, and the crosslinking concentration was also an important parameter not only for the formation of the microspheres, but also for their swelling capacity [10].

\subsection{Chemical Characterization of XBM and XBM-Ca by ATR-FTIR}

To better understand the chemical interactions between the polymeric matrix and the 5-ASA, the ATR-IR was performed. Figure 6A shows the ATR-IR spectra of xylan (I), XBM (II), and PEG (III), while Figure 6B depicted the ATR-IR spectra of XBM-Ca-F9 without 5-ASA (I), XBM-Ca-F9-loaded with 5-ASA (II), and pure 5-ASA (III).
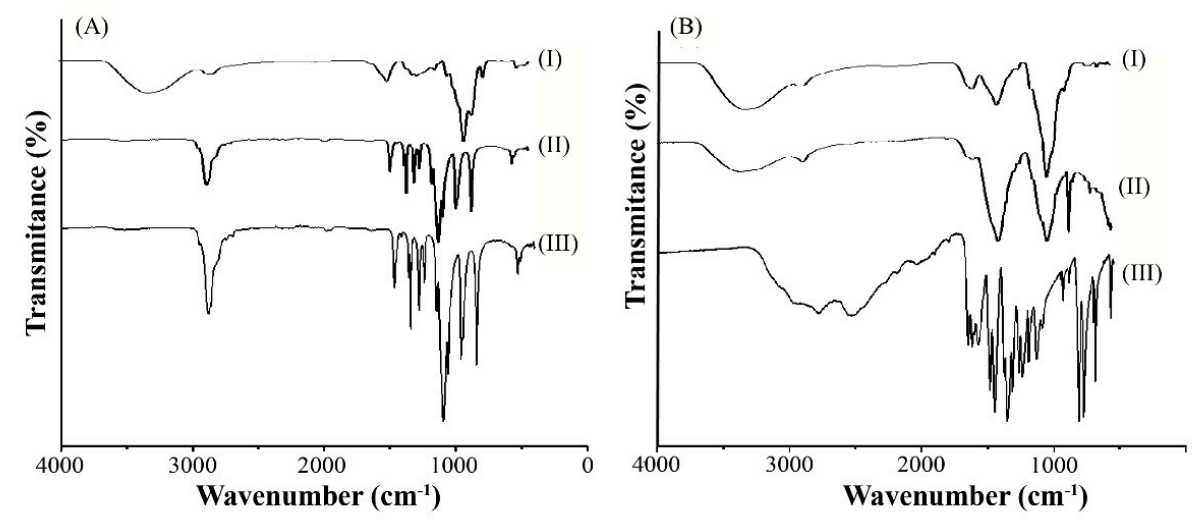

Figure 6. ATR-IR spectra (A) of xylan (I), XBM (II), and PEG (III) and (B) formulation XBM-Ca-F9 without drug (I), XBM-Ca-F9 loaded with 5-ASA (II), and 5-ASA alone (III).

The xylan infrared spectrum was like other spectra observed elsewhere (Figure 6A (I)) [26,27]. Furthermore, it was observed that the spectrum of XBM and PEG (Figure 6A (II and III, respectively)) had similar peaks, likely due to the shielding effect presented by the PEG in the formulation. In fact, after the drying process, it could be possible that the PEG shielding effect form a film and prevent ATR-IR from analyzing XBM. On the other hand, in formulations containing $\mathrm{CaCl}_{2}$ (Figure $6 \mathrm{~B}$ (I and II)), this phenomenon was not observed. It could be speculated that, likely, the $\mathrm{CaCl}_{2}$ play an important role at the interface of the microparticles, which hinders the shielding effect of PEG.

The ATR-IR results also revealed that the bond $\mathrm{P}=\mathrm{O}$ formed during the crosslinking process between the STMP and the polysaccharides was not observed. This phenomenon was equally observed for the empty and loaded XBM-Ca-F9 and might reveal a low degree of crosslinking, which was suggested by Li and co-workers (2012) [15]. The presence of a sharp peak at the $848 \mathrm{~cm}^{-1}$ region was observed for the XBM-Ca-F9 loaded with 5-ASA (Figure 6B (II)), while it was not seen on the empty XBM-Ca-F9 (Figure 6B (I)). This peak is commonly attributed to the C-H bonds of aromatic groups, such as the aromatic ring presented at the 5-ASA structure and was also observed on its spectrum 
in Figure 6B (III) [22]. In addition, the increase on the intensity of the peak near $1500 \mathrm{~cm}^{-1}$ may be associated with the enhancement of drug 5-ASA loading, once these peaks are associated to the $\mathrm{C}-\mathrm{C}$ and $\mathrm{C}=\mathrm{C}$ stretching mode, and also to the $\mathrm{N}-\mathrm{H}$ bond [22].

\subsection{XRD Analysis}

As reported in the literature, xylan XRD analysis (Figure 7 (II)) revealed the profile of an amorphous polymer [26]. Likewise, the diffractogram of the unloaded XBM-Ca-F9 (Figure 7 (III)) also revealed an amorphous structure. This result is very different from the previous reports of crosslinked xylan microparticles prepared with terephthaloyl chloride. However, this is commonly seen in STMP crosslinking products [28]. On the other hand, the diffractogram of 5-ASA alone (Figure 7 (IV)) showed very pronounced peaks at $22.5^{\circ}, 30.6^{\circ}$, and $40.6^{\circ}$, which was also identified in XBM-Ca-F9 loaded with 5-ASA (Figure 7 (I)) XRD analysis. The presence of the peaks from 5-ASA on the XBM-Ca-F9 diffractogram reveals the association of the 5-ASA with the xylan through physical interactions. Once no new bound was identified in the ATR-IR analysis, there was only an enhancement of the peaks at the region between 1400 to $1600 \mathrm{~cm}^{-1}$, as previously discussed.

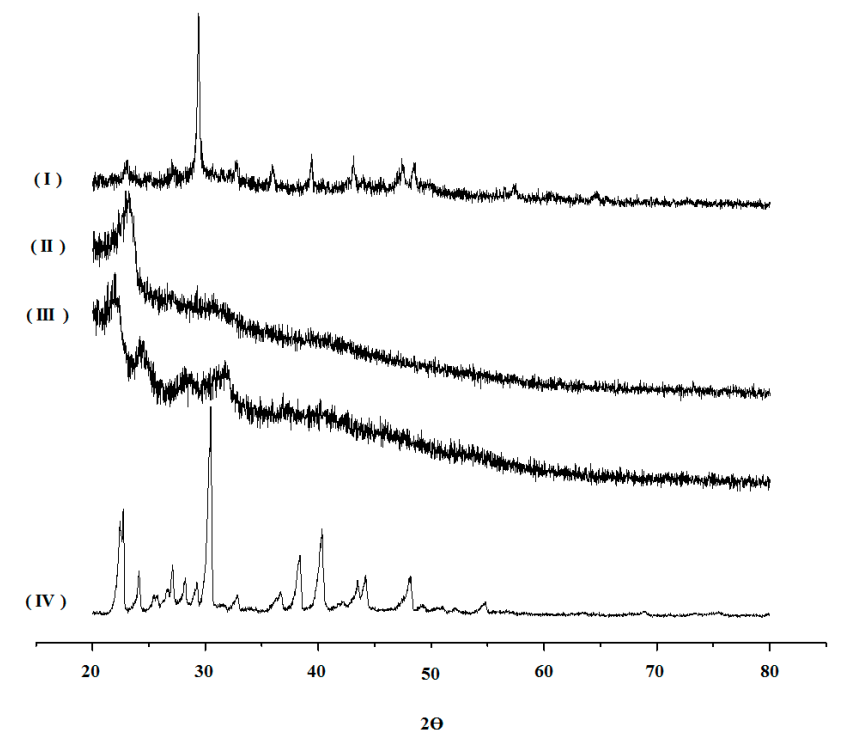

Figure 7. X-ray diffractograms of pure 5-ASA (IV), Formulation XBM-Ca-F9 without drug (III), xylan (II), and Formulation XBM-Ca-F9 loaded with 5-ASA (I).

\subsection{In Vitro Drug Release and Mathematical Modeling}

From the developed formulations shown in Table 1, the formulation XBM-Ca-F9 presented the highest EE\% and, therefore, was used as a model in the study of drug release. According to Figure 8, approximately half of the initial loading of 5-ASA was released into the dissolution medium in approximately $4 \mathrm{~h}$. In fact, despite the initial release, the amount released after $12 \mathrm{~h}$ of the experiment reached $60 \%$. This result indicates that the formulation XBM-Ca-F9 might be able to reach the large intestine with approximately $40 \%$ of its initial loading in 5-ASA, which was later confirmed $(36.4 \% \pm 1.1 \%)$ by the extraction of the 5-ASA from the matrix after $12 \mathrm{~h}$.

This initial release observed in the beginning of the experiment may happen due to the hydration and relaxation of the xylan chains that allow the drug diffusion [29]. However, the ability to hold $40 \%$ of its initial load after $4 \mathrm{~h}$ might be enough for the local treatment of the inflammation. In fact, previous studies with successful pharmacological response carried out with microgranules containing 5-ASA $\left(\right.$ Pentasa ${ }^{\circledR}$ ) showed that the delivery systems reached the colon region in approximately 4 to $6 \mathrm{~h}$ [30]. The retention of the 5-ASA for such a long period into the XBM-Ca-F9 matrix leads to the hypothesis of the ability to deliver the 5-ASA in the colon region by the XBM-Ca-F9 formulation, since it was for 
xylan conjugates with 5-fluoracil (Sauraj and co-workers (2017)). In this work, the authors performed a dissolution assay using the materials and fluids from rats' gastrointestinal tract [31].

In order to understand the mechanism on the initial release of 5-ASA from XBM-Ca-F9, some mathematical models were applied to the data. Initially, a first attempt was made to fit the data to a concentration independent model (e.g., linear model). However, the drug was not released in a constant rate. Thus, models that considered a non-linear release were tested. The Higuchi, Korsmeyer-Peppas, and Peppas-Shalin models were, then, tried. The Higuchi model showed a poor adjustment to the data, likely due to the non Fickian diffusion of the drug through the polymeric matrix, which will be later discussed. On the other hand, the Korsmeyer-Peppas and the Peppas-Shalin model, which consider the Fickian diffusion and the relaxation of the polymeric chains, were able to best fit the data. Therefore, the Peppas-Sahlin model with t-lag was the one with best fitting parameters, once it considers a lag time for the beginning of the drug release (See Table S1) [29,32]. Thus, according to the predictive model, the main mechanism of the 5-ASA release from the Formulation XBM-Ca-F9 was the relaxation of the xylan chains. In addition, it was seen that the contribution of the Fickian diffusion decreased, while the contribution of the relaxation increased over the time.

The release profile observed for the XBM-Ca-F9 was quite different from the xylan-based particles previously produced by our group. The crosslinked microcapsules prepared by Silva et al. (2013) kept constantly the release of the drug into the buffer media, while the spray-dried formulations completely release the drug once in contact to the medium [9]. Thus, to the best of our knowledge, the xylan-based particles developed had the highest encapsulation efficiency and the best drug release profile for physical encapsulation of 5-ASA.

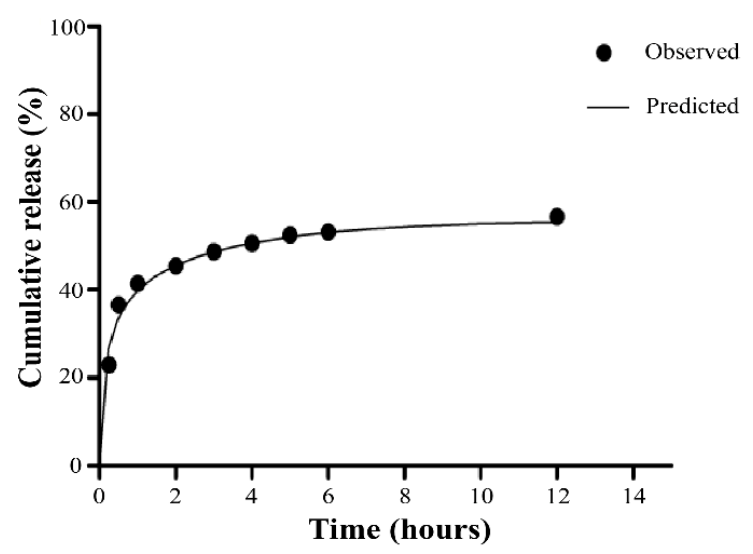

Figure 8. Cumulative drug release profile of the microparticles prepared by the water-in-water emulsion method (Formulation XBM-Ca-F9. Average \pm SD. $n=3$ ).

\section{Conclusions}

The encapsulation of low molecular weight drug using the water-in-water emulsions technique can be achieved using the ionic gelation technique, which is a method to quickly harden the polymeric matrix. Additionally, the addition of $\mathrm{CaCl}_{2}$ did not hinder the assembling of the xylan polymeric chains. Furthermore, the $\mathrm{CaCl}_{2}$ apparently sharpens the particles' size distribution, and all the microparticles produced were spherical and non-aggregated, which reveals the feasibility of the methodology. The kinetic release of the 5-ASA was linked to the relaxation of the xylan chains. Even though there was a 50\% release, seen in the first $4 \mathrm{~h}$, there was a high amount of the 5-ASA that moved into the microparticles after $12 \mathrm{~h}(36.4 \% \pm 1.1 \%)$. To the best of our knowledge, this was the first time that a low molecular weight drug was successfully encapsulated into microparticles produced by this technique, which broadens the application of this technology. This approach can also be used to enhance the therapeutic efficiency of other small molecules, such as budesonide, metronidazole, and dicyclomine. 
Supplementary Materials: The following are available online at http://www.mdpi.com/2076-3417/9/17/3519/s1. Table S1: Mathematical modeling of the experimental data, their equations and statistical parameters.

Author Contributions: B.S.S. performed the experiments, analyzed and discussed the results, and wrote the manuscript. H.R.M. performed the experiments, analyzed and discussed the results, and wrote the manuscript. F.A.J. performed the experiments, analyzed the results, and discussed the results. S.C.C.U. performed the experiments, and analyzed the results. K.C.H.S. performed the experiments. D.C.F.S. contributed to the discussion of the data and edited and revised the manuscript. E.S.T.E. supervised the research work, received the funding, contributed to the discussion of the data, and edited and revised the manuscript.

Funding: The Coordenação de Aperfeiçoamento de Pessoal de Nível Superior (CAPES) Finance code 001 and the Conselho Nacional de Desenvolvimento Científico e Tecnológico (CNPq) funded this research.

Acknowledgments: The authors are grateful to CAPES and CNPq for the financial support offered for this research and to Julieta Genre and Lucas Amaral-Machado, for their discussions when writing this manuscript. In addition, the authors also thank the laboratory of molecular sieves (LABPEMOL) for providing infrared analysis.

Conflicts of Interest: The authors declare no conflict of interest.

\section{References}

1. Marcelino, H.R.; da Silva, A.E.; Gomes, M.C.S.; Oliveira, E.E.; Nagashima, T., Jr.; Pinheiro, G.S.; Silva, A.E.; Timoteo, A.R.S.; Agnez-Lima, L.F.; Ayala, A.P.; et al. Leads from Physical, Chemical, and Thermal Characterization on Cytotoxic Effects of Xylan-Based Microparticles. Polymers (Basel) 2015, 7, $2304-2315$. [CrossRef]

2. Ferreira, I.S.; Bettencourt, A.; Betrisey, B.; Goncalves, L.M.D.; Trampuz, A.; Almeida, A.J. Improvement of the antibacterial activity of daptomycin-loaded polymeric microparticles by Eudragit RL 100: An assessment by isothermal microcalorimetry. Int. J. Pharm. 2015, 485, 171-182. [CrossRef]

3. Liu, M.; Zhong, X.; Yang, Z. Chitosan functionalized nanocochleates for enhanced oral absorption of cyclosporine A. Sci. Rep. 2017, 7, 41322. [CrossRef]

4. Furst, W.; Banerjee, A. Release of Glutaraldehyde From an Albumin-Glutaraldehyde Tissue Adhesive Causes Significant in vitro and in vivo Toxicity. Ann. Thorac. Surg. 2005, 79, 1522-1529. [CrossRef]

5. Nicolai, T.; Murray, B. Particle stabilized water in water emulsions. Food Hydrocoll. 2017, 68, 157-163. [CrossRef]

6. Frith, W.J. Mixed biopolymer aqueous solutions-Phase behaviour and rheology. Adv. Colloid Interface Sci. 2010, 161, 48-60. [CrossRef]

7. Naidu, D.S.; Hlangothi, S.P.; John, M.J. Bio-based products from xylan: A review. Carbohydr. Polym. 2018, 179, 28-41. [CrossRef]

8. Nagashima, T., Jr.; Oliveira, E.E.; Silva, A.E.; Marcelino, H.R.; Gomes, M.C.S.; Aguiar, L.M.; Araújo, I.B.; Soares, L.A.L.; Oliveira, A.G.; Egito, E.S.T. Influence of the lipophilic external phase composition on the preparation and characterization of xylan microcapsules-A Technical Note. AAPS Pharm. Sci. Tech. 2008, 9, 814-817. [CrossRef]

9. Silva, A.E.; Oliveira, E.E.; Gomes, M.C.S.; Marcelino, H.R.; Silva, K.C.; Souza, B.S.; Nagashima, T., Jr.; Ayala, A.P.; Oliveira, A.G.; Egito, E.S.T. Producing xylan/Eudragit(R) S100-based microparticles by chemical and physico-mechanical approaches as carriers for 5-aminosalicylic acid. J. Microencapsul. 2013, 30, 787-795. [CrossRef]

10. Urtiga, S.C.C.; Gabi, C.A.A.L.; Eleamen, G.R.A.; Souza, B.S.; Pessoa, H.L.F.; Marcelino, H.R.; Mendonça, E.A.M.; Egito, E.S.T.; Oliveira, E.E. Preparation and characterization of safe microparticles based on xylan. Drug Dev. Ind. Pharm. 2017, 43, 1601-1609. [CrossRef]

11. Esquena, J. Water-in-water (W/W) emulsions. Curr. Opin. Colloid Interface Sci. 2016, 25, 109-119. [CrossRef]

12. Franssen, O.; Hennink, W.E. A novel preparation method for polymeric microparticles without the use of organic solvents. Int. J. Pharm. 1998, 168, 1-7. [CrossRef]

13. Shum, H.C.; Varnell, J.; Weitz, D.A. Microfluidic fabrication of water-in-water (w/w) jets and emulsions. Biomicrofluidics 2012, 6, 12808-128089.

14. Franssen, O.; Stenekes, R.J.H.; Hennink, W.E. Controlled release of a model protein from enzymatically degrading dextran microspheres. J. Control. Release 1999, 59, 219-228. [CrossRef] 
15. Li, B.-Z.; Wang, L.-J.; Li, D.; Adhikari, B.; Mao, Z.-H. Preparation and characterization of crosslinked starch microspheres using a two-stage water-in-water emulsion method. Carbohydr. Polym. 2012, 88, 912-916. [CrossRef]

16. Freitas, R.A.; Nicolai, T.; Chassenieux, C.; Benyahia, L. Stabilization of water-in-water emulsions by polysaccharide-coated protein particles. Langmuir 2016, 32, 1227-1232. [CrossRef]

17. Hasan, A.S.; Socha, M.; Lamprecht, A.; Ghazouani, F.E.; Sapin, A.; Hoffman, M.; Maincent, P.; Ubrich, N. Effect of the microencapsulation of nanoparticles on the reduction of burst release. Int. J. Pharm. 2007, 344, 53-61. [CrossRef]

18. Zhang, Y.; Huo, M.; Zhou, J.; Zou, A.; Li, W.; Yao, C.; Xie, S. DDSolver: An add-in program for modeling and comparison of drug dissolution profiles. AAPS J. 2010, 12, 263-271. [CrossRef]

19. Zhang, J.; Hwang, J.; Antonietti, M.; Schmidt, B.V.K.J. Water-in-water pickering emulsion stabilized by polydopamine particles and cross-linking. Biomacromolecules 2019, 20, 204-211. [CrossRef]

20. Stenekes, R.J.H.; Hennink, W.E. Equilibrium water content of microspheres based on cross-linked dextran. Int. J. Pharm. 1999, 189, 131-135. [CrossRef]

21. Ham, M.; Moss, A.C. Mesalamine in the treatment and maintenance of remission of ulcerative colitis. Expert Rev. Clin. Pharmacol. 2012, 5, 113-123. [CrossRef]

22. Dash, A.K.; Brittain, H.G. Mesalamine. In Analytical Profiles of Drug Substances and Excipients; Academic Press: Cambridge, MA, USA, 1998; Volume 25, pp. 209-242.

23. Koetting, M.C.; Guido, J.F.; Gupta, M.; Zhang, A.; Peppas, N.A. pH-responsive and enzymatically-responsive hydrogel microparticles for the oral delivery of therapeutic proteins: Effects of protein size, crosslinking density, and hydrogel degradation on protein delivery. J. Control. Release 2016, 221, 18-25. [CrossRef]

24. Nguyen, M.-H.; Tran, T.-T.; Hadinoto, K. Controlling the burst release of amorphous drug-polysaccharide nanoparticle complex via crosslinking of the polysaccharide chains. Eur. J. Pharm. Biopharm. 2016, 104, 156-163. [CrossRef]

25. Li, B.-Z.; Wang, L.-J.; Li, D.; Chiu, Y.L.; Zhang, Z.-J.; Shi, J.; Chen, X.D.; Mao, Z.-H. Physical properties and loading capacity of starch-based microparticles crosslinked with trisodium trimetaphosphate. J. Food Eng. 2009, 92, 255-260. [CrossRef]

26. Oliveira, E.E.; Silva, A.E.; Nagashima, T., Jr.; Gomes, M.C.S.; Aguiar, L.M.; Marcelino, H.R.; Araujo, I.B.; Bayer, M.P.; Ricardo, N.M.P.S.; Oliveira, A.G.; et al. Xylan from corn cobs, a promising polymer for drug delivery: Production and characterization. Bioresour. Technol. 2010, 101, 5402-5406. [CrossRef]

27. Gao, C.; Ren, J.; Zhao, C.; Kong, W.; Dai, Q.; Chen, Q.; Liu, C.; Sun, R. Xylan-based temperature/pH sensitive hydrogels for drug controlled release. Carbohydr. Polym. 2016, 151, 189-197. [CrossRef]

28. Li, B.-Z.; Wang, L.-J.; Li, D.; Bhandari, B.; Li, S.-J.; Lan, Y.; Chen, X.D.; Mao, Z.-H. Fabrication of starch-based microparticles by an emulsification-crosslinking method. J. Food Eng. 2009, 92, 250-254. [CrossRef]

29. Brazel, C.S.; Peppas, N.A. Mechanisms of solute and drug transport in relaxing, swellable, hydrophilic glassy polymers. Polymer (Guildf) 1999, 40, 3383-3398. [CrossRef]

30. Wilding, I.R.; Kenyon, C.J.; Hooper, G. Gastrointestinal spread of oral prolonged-release mesalazine microgranules (Pentasa) dosed as either tablets or sachet. Aliment. Pharmacol. Ther. 2000, 14, 163-169. [CrossRef]

31. Kumar, S.U.; Gopinath, P.; Negi, Y.S. Synthesis and bio-evaluation of xylan-5-fluorouracil-1-acetic acid conjugates as prodrugs for colon cancer treatment. Carbohydr. Polym. 2017, 157, 1442-1450. [CrossRef]

32. Peppas, N.A.; Sahlin, J.J. A simple equation for the description of solute release. III. Coupling of diffusion and relaxation. Int. J. Pharm. 1989, 57, 169-172. [CrossRef]

(C) 2019 by the authors. Licensee MDPI, Basel, Switzerland. This article is an open access article distributed under the terms and conditions of the Creative Commons Attribution (CC BY) license (http://creativecommons.org/licenses/by/4.0/). 